\title{
AIDS PREVENTION IN SOUTH AFRICA
}

\author{
Sinead Delany-Moretlwe ${ }^{1 \star}$, Glenda Gray ${ }^{2 \star}$, Ashraf Kagee ${ }^{3 \star}$, Landon Myer ${ }^{4 \star}$, \\ Adrian Puren ${ }^{6 \star}$, Gita Ramjee ${ }^{7 \star}$, Helen Rees ${ }^{1 \star}$, Leslie Swartz ${ }^{3 \star}$, Linda-Gail Bekker
}

More individuals were newly infected with HIV in 2005 than any other year. Sub-Saharan Africa and especially southern Africa bears the brunt of this pandemic. Although the picture in sub-Saharan Africa is largely one of a 'stable' epidemic where AIDSrelated mortality is matched by the incidence of new infections, some countries in the Southern regions have continued to see increasing HIV prevalence. ' In this light, there is an urgent need for new approaches to HIV prevention. Here we review the current state of HIV prevention technologies, with particular emphasis on new approaches to HIV prevention that have particular promise in southern Africa. The focus here is on interventions that address sexually transmitted HIV, since the vast majority of new HIV infections in Africa are through heterosexual contact, and other important HIV prevention interventions (such as blood safety interventions and the prevention of mother-to-child transmission) are not included.

\section{LESSONS FROM HIV EPIDEMIOLOGY IN AFRICA}

Our understanding of prevention interventions can be framed by two general principles from infectious diseases epidemiology. First, the transmission dynamics of HIV can be described in terms of the basic reproductive number (Ro), which represents the number of secondary infections emanating from a singe infectious individual (i.e. a primary case) introduced into a population of susceptible individuals. The equation: $R o=B C D$ shows how the basic reproduction number Ro is influenced by the probability of HIV transmission between individuals ( $B$, a function of both the infectiousness of the primary case and the susceptibility of uninfected individuals), as well as the number of sexual partners (c) and the duration of infectiousness (D) of the primary case. ${ }^{2}$ Evidence from different parts of Africa suggests that variability in both the transmission probability and sexual partner changes are important in explaining the variable course of HIV epidemics in different regions.

The second key HIV prevention principle suggested by infectious diseases epidemiology is that reductions in transmission risk among the most sexually active members of the population can have a disproportionately large impact on the HIV epidemic. In other words, the most efficient and effective prevention strategies should be targeted at specific population groups where HIV acquisition risk is high.

Recent epidemiological research demonstrates how these principles may operate in sub-Saharan Africa. At a national level, Zimbabwe is one of the few countries that has seen a marked decrease in HIV prevalence that can be linked to changes in sexual behaviours (other notable examples include

Reproductive Health \& HIV Research Unit ${ }^{1}$ and Perinatal HIV Research Unit ${ }^{2}$, University of the Witwatersrand; Department of Psychology, Stellenbosch University ${ }^{3}$; Infectious Diseases Epidemiology Unit, School of Public Health \& Family Medicine ${ }^{4}$, and Desmond Tutu HIV Centre, Institute of Infectious Diseases \& Molecular Medicine ${ }^{5}$, University of Cape Town; National Institute of Communicable Diseases ${ }^{6}$; and HIV Prevention Research Unit, Medical Research Council?.

*Authors are listed alphabetically.

THE SOUTHERN AFRICAN JOURNAL OF HIV MEOICINE
Uganda and Thailand). One recent analysis from Zimbabwe, ${ }^{3}$ based on repeated studies of a rural Zimbabwean population between 1998 and 2003, shows a 23\% reduction in HIV prevalence among men aged $17-29$ years and $49 \%$ reduction among young women aged $15-24$ years. These reductions have been attributed to proportional decline in sexual risk behaviours, namely delayed sexual debut, reduction in numbers of casual sexual partners and more condom use among females.

Further insights into the population spread of HIV in Africa come from the Four Cities study, ${ }_{1}$ a cross-sectional study of HIV, other sexually transmitted infections, and sexual behaviours from Kisumu (Kenya), Ndola (Zambia), Cotonou (Benin) and Yaounde (Cameroon). This study suggested that high male circumcision rates in West Africa may have reduced the rate of HIV and other STIS, while genital ulcer disease, especially herpes simplex virus type 2 (HSV-2), may have contributed to higher rates in East and Southern Africa.

\section{MALE CIRCUMCISION}

The role of male circumcision in the prevention of HIV and STI acquisition became a hotly debated topic when a number of cross-sectional studies from different parts of Africa suggested that this intervention might have a protective effect, ${ }^{5,6}$ and this finding has been supported by more recent data suggesting reductions in HIV transmission among HIV serodiscordant couples where the man is circumcised. ${ }^{7}$ In addition to these epidemiological data, there are biologically plausible mechanisms for the observed reduction in HIV-1 acquisiton among circumcised males. The inner mucosa of the foreskin is rich in HIV-1 target cells, e.g. dendritic cells, CD4+ T cells, and macrophages that express relevant HIV-1 binding receptors such as chemokine receptors (CCR5) and DC-SIGN. ${ }^{8}$ By contrast the external foreskin is keratinised and much less vulnerable to HIV infection. After circumcision, the only exposed mucosa is in the urethral meatus. Removal of the foreskin's target cells and receptors can represent a direct biological mechanism of protection.

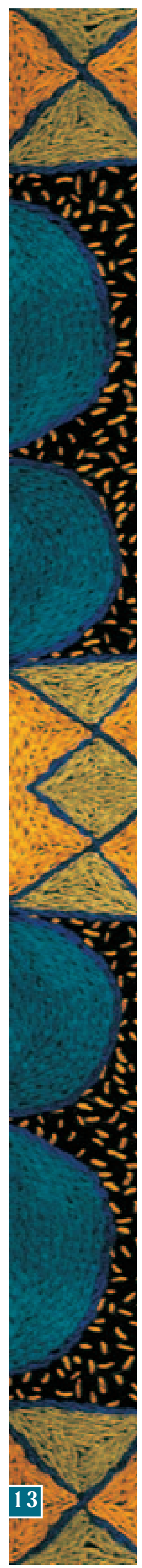


Three randomised controlled trials (RCTs) have recently been initiated in sub-Saharan Africa to explore the efficacy of male circumcision. The results of the RCT conducted in Orange Farm, South Africa, demonstrated a 60\% reduction in the risk of acquiring HIV infection over 21 months of follow-up in newly circumcised, 18 - 24-year-old males. The incidence rate in the intervention group was 0.7 per 100 person years while in the control group it was 2.2 per 100 person years, a crude incidence rate ratio of 0.35 (95\% Cl: $0.20,0.60)$. The other two RCTs (in Uganda and Kenya) are still to be completed. Should these randomised controlled trials also show the efficacy of male circumcision in reducing HIV acquisition risk, the implementation of this modality in the general population will still require further investigation of a range of issues including acceptability, rates and types of adverse events of the procedure, cost and logistics of introduction, and importantly the long-term effect of circumcision on behaviours such as partner reduction and condom use. ${ }^{9}$

\section{VACCINES}

An effective preventive HIV vaccine would provide the best method for controlling the HIV pandemic, especially in underresourced countries, and so any successful vaccine will need to be applicable to all regions regardless of virus subtype and be licensed for use in all age groups including older children and adolescents. After almost 20 years of HIV vaccine clinical trials, and numerous phase 1 and 2 studies, there are a variety of vaccine products in the pipeline that look hopeful enough to be subjected to efficacy trials. ${ }^{10}$

\section{VACCINE STRATEGIES}

For a number of years the vaccine pipeline was limited to simple gp 120 or gp 160 proteins based on lab strains of the virus, synthetic peptides and simple poxvirus HIV-recombinant vectors. The constructs currently in the pipeline include gp 120 constructs based on clinical HIV isolates, bird virus vectors and other vectors such as modified vaccinia Ankara and Venezuelan equine encephalitis virus replicon expressing multiple HIV genes, and different constructs of naked DNA. The most promising candidates in the last few years have included live attenuated vectors containing a variety of HIV genes. These attenuated vectors include adenovirus, associated adenovirus and sindbis. ${ }^{11,12}$

\section{CHALLENGES TO VACCINE DESIGN}

The lack of an immune correlate of protection (clear type of immune response needed to provide protection against HIV) and the lack of an adequate animal model that can mimic human disease infection poses significant scientific challenges to developing a successful vaccine. In addition HIV-1 genetic diversity may complicate the development of a globally relevant HIV vaccine. Diversity may be addressed by the development of vaccine candidates comprised of cocktails of proteins of regional variants with the assumption that the immune responses elicited by a multiclade, multi-gene vaccine will be of sufficient cross-reactivity to protect against a range of wild-type strains. ${ }^{13}$

\section{PROTECTION REQUIRED}

Current HIV vaccine candidates elicit reasonably potent cellular immune responses, but very low levels of neutralising antibodies. Cytotoxic T-lymphocytes are part of the cellular immune response that controls viral replication. Vaccines that produce strong CTL responses are more likely to control viral replication and thus reduce viral load. Used as preventive vaccines they may then modify disease in an individual who has breakthrough infection, leading to less morbidity, longer time to AIDS and possibly less HIV transmission. Antibodies provide the first line to the immune system defence and neutralising antibodies inactivate or prevent the virus making contact with target cells, providing the best possibility to abort or prevent infection. Neutralising antibody stimulation would thus be a highly sought after characteristic in a preventive vaccine, but has to date been very difficult to achieve. The gp 160 protein on the outside of the virus is important for stimulating neutralising antibodies but is oligomeric and susceptible to glycosylation. Most HIV infections occur via the mucosal route and so it may be important to stimulate mucosal immunity in rectal and genital tracts as well as cellular and humoral responses in the blood to achieve protection. ${ }^{10,14}$

Prime-boosting is a new combination strategy that seeks to enhance vaccine responses by invoking various types of immunity. A typical strategy would involve priming with a naked DNA vaccine which would be expected to do little more than stimulate production of memory $T$ cells, followed by boosting with a live vector/protein, which would then stimulate a strong cellular response as well as neutralising antibodies. So far early studies in humans have shown this to be safe. ${ }^{12}$

\section{PROMISING CANDIDATES THAT ARE FURTHER ON IN HUMAN TESTING}

A number of world-wide networks, e.g. the HIV Vaccine Trials Network (HVTN), the International AIDS Vaccine Initiative and the South African AIDS Vaccine Initiative, as well as pharmaceutical companies, are involved in the global effort to develop and test preventive vaccines. After 20 years of ongoing research and only one completed, albeit ineffective, phase 3 trial in AIDSVAX, a bivalent recombinant gp120 vaccine, the HIV vaccine development pipeline is full with a number of promising candidates that look good enough to move beyond phase 1 and 2 clinical trials into efficacy testing. The NIAID in collaboration with Merck and the HVTN have initiated a test of concept study (phase Ilb) in North America and South America, the Carribean and Australia. This randomised placebo-controlled trial will test Merck's HIV vaccine, the MRK Ad5 HIV-1 gag/pol/nef vaccin, in a study that will enroll 3000 participants. The trial is set to answer two questions: whether (i) pre-existing immunity to the adenovirus subtype 5 (Ad5), which is the vector used in this vaccine design, dampens the immune response to this vaccine; and (ii) the cellular immune response elicited by this vaccine is robust enough to protect against infection. This trial will also assess disease progression in those who become infected despite receiving the vaccine.

A sister trial, the HVTN 503, is scheduled to take place in South Africa later in 2006. Since South Africa is a subtype $C$ region 
where there is a high prevalence of pre-existing immunity to Ad5, this trial will answer the question regarding the importance of sub-type in vaccine design as well as the role of pre-existing immunity to Ad5. Three thousand adults, the majority of whom are likely to be women, will participate in this trial.

Shortly thereafter, a second large efficacy trial is planned to evaluate the VRC multiclade DNA vaccine and the VRC multiclade Ad5-based vaccine sequentially also in a primeboost strategy.

\section{SAAVI VACCINE DEVELOPMENT PROGRAMME}

The South African AIDS Vaccine Initiative (SAAVI) is also contributing significantly to the global effort. Not only are there several community clinical trial sites being prepared around the country to ensure that there are large numbers of well-educated and carefully prepared healthy volunteers to participate in these imminent large-scale trials, but in collaboration with the University of Cape Town there are also two products that are about to enter into human testing. The SAAVI DNA-C2 is a multigene subtype-C DNA vaccine and the SAAVI MVA-C is a multigene HIV-1 subtype-C recombinant $M V A$ vaccine. These two South African vaccine candidates will be tested in a 'prime-boost' phase I trial design in collaboration with the HVTN both in South Africa and the USA in 2007.

\section{ADOLESCENT INVOLVEMENT IN VACCINE TRIALS}

One of the most effective ways to curb the epidemic in the developing world would be to vaccinate older children and adolescents prior to their sexual debut. The use of an HIV vaccine in this population will require clinical trials in adolescents to determine the vaccine's safety and immunogenicity. A precise strategy for the involvement of adolescents in clinical trials is urgently required, and again SAAVI and South African researchers are leaders in the international dialogue on this topic. ${ }^{15,16}$

\section{VACCINE MANUFACTURE, PROCUREMENT AND DISTRIBUTION}

Previous vaccine history has shown that once an efficacious vaccine is developed the usual pattern of deployment is in the developed world first and the developing world later. A plan for the urgent procurement and distribution of a successful HIV vaccine when available needs to exist to make the vaccine available it is where needed most - namely in resource-poor settings - as quickly as possible. This includes mechanisms for pricing, global financing, demand estimates and preparedness for production capacity, appropriate delivery systems and strategies for high-risk populations. Harmonisation of African regulatory systems and guidelines for approval are urgent, not only for this later phase of vaccine deployment, but also during this current important phase of vaccine testing. The newly established HIV Vaccine Enterprise aims to co-ordinate global efforts around these issues. ${ }^{17}$

\section{MICROBICIDES}

In view of the problems associated with male condoms, developing HIV prevention technologies that are under the control of women is an important avenue for HIV prevention. One of the most promising technologies currently in largescale human trials is vaginal microbicides. A microbicide is a substance formulated to significantly reduce transmission of HIV and other sexually transmitted infections (STIs) when applied topically to the vagina or rectum. ${ }^{18,19}$ They can be formulated as gels, creams, films, suppositories, sponges or vaginal rings, or used in conjunction with other barrier methods such as the diaphragm or cervical cap. The mode of action of microbicides includes antiviral activities, barrier action between the pathogens and vaginal and rectal tissue, or modification of vaginal or rectal milieu which makes HIV infection less likely. ${ }^{20}$ Compared with male or female condoms, microbicides are expected to interfere less with intimacy and sexual pleasure and be more discreet. ${ }^{21}$

Although microbicides are primarily being developed for use by women, it is possible that they may have a bidirectional protective effect for men as well. As women's reproductive intentions alter throughout their lives, both contraceptive and non- contraceptive microbicides are being developed. Another research agenda is to develop a microbicide that is protective for partners practising anal sex, as such a product could be used for both heterosexual and MSM sex. Importantly, a microbicide shown to be safe and effective should be easily available over the counter. Mathematical modelling of a microbicide assumed to be 60\% efficacious and to have 20\% uptake by women at risk of HIV suggests that 2.5 million new HIV infections could be averted in developing countries.

There are currently 16 candidate microbicides in clinical development including five products in advanced stages of clinical testing (phase IIB/III trials). All of these products are being tested in Africa, and most are being tested in South Africa specifically. More than 20000 women will take part in the current trials over the next 5 years. Results of the first products are expected to be available in late $2008 .{ }^{12,22}$

\section{BARRIER METHODS}

Currently the most widely available tool for prevention of HIV infection during sexual intercourse is the male condom. Male condoms afford a high degree of protection: consistent and correct male condom use reduces HIV transmission by between $80 \%$ and 97\%. However, in many parts of Africa condoms are not acceptable as they act as a contraceptive and may also interfere with sexual pleasure and reduce intimacy. Men predominantly control use of male condoms during sexual intercourse, and many women do not have the power to negotiate condom use in their relationships. As a result, there have been significant recent developments in other types of barrier methods to prevent the sexual transmission of $\mathrm{HIV}^{23}$

\section{THE FEMALE CONDOM}

Numerous studies have shown that the female condom is an acceptable method for many women and men, and is a valuable alternative for women whose partners refuse to use male condoms. Unlike the male condom, the female condom can be inserted some time before sex, and does not depend on the same degree of male co-operation for its successful use. The female condom is a soft, loose-fitting polyurethane 
sheath that covers the vagina, cervix and external genitalia. Laboratory studies have shown that the female condom is effective at preventing the transmission of viruses and bacteria. While there are less clinical data available than for the male condom, the WHO has agreed that the female condom is effective in preventing HIV and other sexually transmitted infections (STIs). However, despite the effectiveness and acceptability of the female condom, they are not widely distributed or available in South Africa. This is partly due to costs (more than 10 times that of a male condom) but also to a lack of commitment to this femaleinitiated technology by donors and governments. Nonetheless, the female condom represents an important HIV prevention option where it is available. . $^{4425}$

\section{CERVICAL BARRIER METHODS}

In the last few years, interest has grown in cervical barrier methods as potential technologies for HIV prevention. The cervix is covered by a single cell layer of columnar epithelium, in contrast to the stratified squamous epithelium of the vagina. As a result cervical columnar epithelium is more friable than the stratified squamous epithelium of the vaginal walls, making it more susceptible to mechanical disruption. This anatomical vulnerability is compounded by the increased presence around the cervix of surface receptors targeted by HIV as well as inflammatory cytokines, both which may also facilitate HIV infection. Additional evidence from primate experiments has shown that cervical epithelium is the first site of infection after vaginal exposure to simian immunodeficiency virus (SIV).

On the basis of this evidence, barrier methods that protect the cervix specifically (such as the diaphragm, the sponge and the cervical cap) may be useful tools for HIV prevention, and there are currently a number of trials of this topic underway in southern Africa. There is ample evidence that the different cervical barrier methods are safe, easy to use, inexpensive and highly acceptable to both women and men. New cervical barriers are in development, and the efficacy of these methods against pregnancy, STIs and HIV is being investigated. In addition, should an effective microbicide be identified, the combination of a cervical barrier and a microbicide may offer even greater potential for prevention of HIV transmission. ${ }^{26}$

\section{HERPETIC GENITAL ULCER DISEASE CONTROL}

The role of bacterial STIs (including syphilis, chlamydia infections and gonorrhoea) in increasing the risk of HIV infection is well established. More recently, HSV-2 has received attention as an important risk factor for heterosexually-transmitted HIV. HSV-2 is the most common sexually transmitted infection worldwide, typically causing recurrent episodes of genital ulcers, although a large proportion of infected individuals are asymptomatic. ${ }^{27-29}$

Recently, there have been growing concerns about the role of HSV-2 in HIV transmission given the fact that it is the most common cause of recurrent genital ulcer disease in a significant proportion of the adult population, which is also at risk for HIV. Genital ulcers act as a portal of entry or exit for HIV and activated lymphocytes, including CD4 cells, are frequently recruited to these sites of inflammation and are primed to receive or present HIV at the site of ulceration. A recent meta-analysis of 19 epidemiological studies showed that prevalent HSV-2 may increase the risk of HIV acquisition in men and in women by as much as 3-fold even after adjustment for sexual behaviour, and that HSV-2 may account for as many as 38 - 60\% of new HIV infections in women, and $8-49 \%$ in men in the general populations. ${ }^{30}$

Controlling HSV-2 may have an important impact on HIV incidence, particularly in settings where HSV-2 prevalence is high. Currently the options for herpes control are limited to a few strategies: primary prevention through condom use and behavioural modification will be useful in uninfected populations, e.g. young people. Treatment of HSV-2, primarily with acyclovir, may also have an effect on HIV and randomised control trials are currently underway to investigate this. There is strong evidence that suppressive treatment for genital herpes reduces the levels of HIV in the genital secretions of infected women (a potential marker of infectiousness and therefore transmission). The results of trials to evaluate the effect of suppressive therapy on HIV acquisition and HIV transmission are likely to be available within 2 years. ${ }^{31}$

\section{SOCIOBEHAVIOURAL INTERVENTIONS}

In addition to the biomedically focused interventions discussed above, behavioural interventions remain a valuable strategy for reducing new infections. We know what specific behaviours contribute to the spread of HIV in South Africa (large numbers of new sexual partners and unprotected sexual contacts), and yet the virus continues to spread. Two theories have been used to explain and predict the spread of HIV and attempts to curb this spread. ${ }^{32}$

The first theory focuses on individual behaviour and behaviour change, and discusses the ways in which intentions, attitudes and beliefs affect health behaviour. The second theory involves understanding broader social issues which affect the epidemic. For example, it seems clear that an important part of what drives the epidemic is not individual intentions and behaviours but broader social, economic and cultural conditions. Women, for example, may find themselves in a situation in which they have little power or decision-making in their lives, and for reasons of immediate survival may be forced into transactional sexual relationships with men that will involve unprotected sex. A key challenge for those wishing to reduce the spread of HIV through behaviour change is to design interventions that bridge the divide between individual models of behaviour change and focus on broader social issues. Health practitioners, by virtue of their training, are often better at thinking about individual level issues and interventions than in intervening and assessing interventions at a broad social level.

The need for both levels does, however, remain. Successes in HIV prevention in African countries such as Uganda, furthermore, have similarly been attributed both to individuallevel interventions and to the commitment of government as a whole to creating social conditions and relationships in which safe sex is not a taboo topic but an issue which is seen to concern everyone. Campbel/ ${ }^{33}$ has discussed the importance of what she terms 'political will' in curbing and containing the epidemic. 
At a practical level, voluntary counselling and testing (VCT) represents an important strategy for changing sexual behaviours. There is substantial evidence that appropriate risk reduction messages provided during VCT can have a significant impact in reducing high risk behaviours among individuals who wish to be tested for HIV. In one multicountry trial, including sites in Kenya and Tanzania, there was a more than 30\% reduction in unprotected intercourse among individuals receiving VCT compared with individuals who received health promotion messages only. This study and related research has contributed to what some policy makers have called a 'serostatus approach' to addressing the HIV epidemic, in which HIV testing is a critical first step that can be used to target services for HIV prevention (for HIV-negative individuals) or care and treatment (for HIV-positive individuals). ${ }^{34}$

Within South Africa, a number of large, multifaceted behaviour change campaigns have been developed to reduce sexual risk behaviours, particularly among young people. Two of the best known of these are Soul City (a multimedia intervention including television, radio and magazines) and LoveLife (a media campaign linked to adolescent-focused health care services). It is difficult to examine the precise impacts of campaigns of this type, but preliminary evaluations have suggested that these interventions have played a valuable role in both increasing awareness and knowledge of HIVIAIDS among individuals, and in shifting popular perceptions of the disease in communities heavily affected by HIV. However the role that these interventions may play in altering the incidence of HIV infection remains unknown. ${ }^{12}$

\section{CONCLUSION}

While prevention efforts need to be redoubled in sub-Saharan Africa, where prevalence rates are so high, there is at least some hope for success.' The most recent UNAIDS report on the Global AIDS epidemic states: 'Among the notable new trends are the recent declines in national HIV prevalence in two subSaharan African countries (Kenya and Zimbabwe), urban areas of Burkina Faso, and similarly in Haiti, in the Caribbean, alongside indications of significant behavioural change including increased condom use, fewer partners and delayed sexual debut.' However, perhaps even more spine-chilling are indications that in regions where HIV rates had declined, a resurgence in new infections particularly among specific risk groups such as young MSM populations, is occurring. A subject we have not covered here is the ultimate impact of antiretroviral treatment (ART) and the impact that the new drive to increase world access to ART will have on transmission and subsequently prevalence in many parts of the world. At the very least, in this new era of antiretroviral access, limiting numbers requiring antiretrovirals in the long term is good medicine and cost saving. This demands more research into efficacious prevention strategies and monitoring vigilance. It has been said that an ounce of prevention is worth a pound of cure ... but it takes a ton of work!

\section{REFERENCES}

1. Asamoah-Odei E, Garcia Calleja JM, Boerma JT. HIV prevalence and trends in subSaharan Africa: no decline and large subregional differences. Lancet 2004; 364: 35.

2. Anderson RM, May RM. Infectious Diseases of Humans: Dynamics and Control. Oxford: Oxford University Press, 1991.
3. Gregson S, Garnett GP, Nyamukapa CA, et al. HIV decline associated with behaviour change in eastern Zimbabwe. Science 2006; 311: 664-666.

4. Buve $A$, Weiss HA, Laga $M$, et al. The epidemiology of trichomoniasis in women in four African cities. AIDS 2001; 15: Suppl 4, S89-S96.

5. Auvert B, Taljaard D, Lagarde E, Sobngwi-Tambekou J, Sitta R, Puren A Randomized, controlled intervention trial of male circumcision for reduction of HIV infection risk: The ANRS 1265 Trial. PLoS Med 2005; 2(11): e298.

6. Scott BE, Weiss HA, Viljoen JI. (2005) The acceptability of male circumcision as an HIV intervention among a rural Zulu population, KwaZulu-Natal, South Africa. AIDS Care 2005; 17: 304-313.

7. Gray RH, Kiwanuka N, Quinn TC, et al. Male circumcision and HIV acquisition and transmission: Cohort studies in Rakai, Uganda. Rakai Project Team. AIDS 14: 2371-2381

8. Patterson BK, Landay $A$, Siegel $J N_{\text {, }}$ et al. Susceptibility to human immunodeficiency virus-1 infection of human foreskin and cervical tissue grown in explant culture. Am J Pathol 2002; 161: 867-873.

9. Gray R, Azire J, Serwadda D, et al. Male circumcision and the risk of sexually transmitted infections and HIV in Rakai, Uganda. AIDS 2004; 18: 2428-2430.

10. McMichael AJ. HIV vaccines. Annu Rev Immunol 2006; 24: 227-255.

11. Joseph J, Etcheverry F, Alcami J, Maria GJ. A safe, effective and affordable HIV vaccine - an urgent global need. AIDS Rev 2005; 7(3):131-138.

12. Abdool-Karim SS, Abdool-Karim O. HIV/AIDS in South Africa. Cambridge: Cambridge University Press, 2005.

13. Slobod $\mathrm{KS}$, Coleclough $\mathrm{C}$, Bonsignori $\mathrm{M}$, et al. HIV vaccine rationale, design and testing. Curr HIV Res 2005; 3(2): 107-112.

14. Belyakov IM, Ahlers JD, Berzofsky JA. Mucosal AIDS vaccines: current status and future directions. Expert Rev Vaccines 2004; 3(4 Suppl):S65-S73.

15. Jaspan HB, Lawn SD, Safrit JT, Bekker L-G. The maturing immune system: implications for development and testing HIV-1 vaccines for children and adolescents. AIDS 2006; 20(4): 483-494

16. Bekker L-G, Jaspan H, McIntyre J, Wood R, Gray G. Adolescents and HIV Vaccine trials: what are the clinical trial site issues? I Int Assoc Physicians AIDS Care 2005; 4(4): 93-97.

17. Barth-Jones DC, Cheng $H$, Kang LY, et al. Cost effectiveness and delivery study for future HIV vaccines. AIDS 2005; 19(13): w1-w6.

18. Scholand SJ, DeSimone JA, Pomerantz RJ. Anti-HIV-1 microbicides - 'chemical condoms' designed to limit the scourge of the HIV-1 pandemic. Curr Pharm Des 2005; 11(29): 3747-3756.

19. Stone A. Microbicides: A new approach to preventing HIV and other sexually transmitted infections. Nature Review 2002; 1: 977-985.

20. D'Cruz OJ, Uckun FM. Dawn of non-nucleoside inhibitor-based anti-HIV microbicides. J Antimicrob Chemother 2006; 57(3): 411-423. Epub 23 Jan 2006.

21. Orner $\mathrm{P}$, Harries J, Cooper $\mathrm{D}$, Challenges to microbicide introduction in South Africa. Soc Sci Med 2006; 63(4): 968-978.

22. Van Damme L, Ramjee G, Alary M, et al. Effectiveness of COL-1492, a nonoxynol -9 vaginal gel, on HIV transmission in female sex workers: a randomized controlled trial. Lancet 2002; 360: 971-977.

23. Davis KR, Weller SC. The effectiveness of condoms in reducing heterosexual transmission of HIV. Family Planning Perspectives 1999; 31: 272-279.

24. Smit J, Beksinska M, Vijayakumar G, Mabude Z. Short-term acceptability of the Reality polyurethane female condom and a synthetic latex prototype: a randomized crossover trial among South African women. Contraception 2006; 73(4): 394-398. Epub 3 Feb 2006.

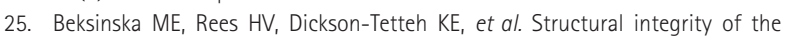
female condom after multiple uses, washing, drying, and re-lubrication. Contraception 2001; 63: 33-36.

26. Warren $M$, Philpott A. Expanding safer sex options: introducing the female condom into national programmes. Reprod Health Matters 2003; 11(21): 130 139.

27. Sacks SL, Griffiths PD, Corey L, et al. HSV-2 transmission. Antiviral Res 2004 63: Suppl 1: S27-S35.

28. Ramjee G, Williams B, Gouws E. The impact of incident and prevalent herpes simplex virus-2 infection on the incidence of HIV-1 infection among commercial sex workers in South Africa. J Acquir Immune Defic Syndr 2005: 39(3): 333-339.

29. Wasserheit J. Epidemiologic synergy: interrelationships between human immunodeficiency virus infection and other sexually transmiited diseases. Sex Transm Dis 1992; 19: 61-77.

30. Freeman $\mathrm{EE}$, Weiss $\mathrm{HA}$, Glynn JR. Herpes simplex virus 2 infection increases HIV acquisition in men and women: systematic review and meta-analysis of longitudinal studies. AIDS 2006; 20(1): 73-83

31. Celum $C$, Levine $R$, Weaver $M$, Wald $A$. Genital herpes and human immunodeficiency virus: double trouble. Bull World Health Organ 2004; 82(6): 447-453.

32. Aaro LE, Flisher AJ, Kaaya S, et al. Promoting sexual and reproductive health in early adolescence in South Africa and Tanzania: development of a theory- and evidence-based intervention programme. Scand J Public Health 2006: 34(2):150-158

33. Campbell C. Letting Them Die: Why HIV/AIDS Prevention Programmes Fail. Oxford: James Currey, Bloomington \& Indianapolis: Indiana University Press, and Cape Town: Double Story/Juta, 2003.

34. Urassa $W$, Kaaya $S$, Mwakagile $D$, et al. Evidence of a substantial decline in prevalence of HIV-1 infection among pregnant women: Data from 1995 to 2003 in Dar es Salaam, Tanzania. Scand J Public Health 2006; 34(3): 272-278.

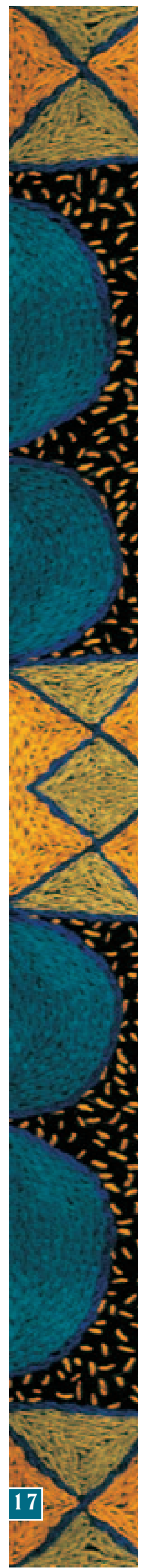

